\title{
Do Gene and Cell Therapies will Ultimately Replace Repeated Injections for the Treatment of Chronic Diseases
}

\author{
Shravana Kumar Chinnikatti* \\ Consultant Oncologist, Salmaniya Medical Complex, Bahrain \\ *Corresponding author: Shravana Kumar Chinnikatti, Consultant Oncologist, Salmaniya Medical Complex, Bahrain, Email: shravanonco@gmail.com

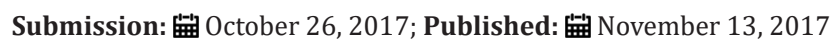

\section{Opinion}

Medical innovations are extremely important to be protected and exploited in an effective manner as they have strong social and economic impact. The potential of the gene editing tool CRISPR (Clustered Regularly Interspaced Short Palindromic Repeats) phenomenon seems to be the latest experimental use of the technology is creating skin grafts that trigger the release of insulin and help manage diabetes. This comes from the Research that has successfully tested the idea with mice that gained less weight and showed a reversed resistance to insulin because of the grafts [1]. I think this approach could eventually be used to treat a variety of metabolic and genetic conditions, not just diabetes it's a question of using skin cells to trigger different chemical reactions in the body because easy and abundantly available skin cells available at our disposal. This CRISPER is a new and innovative way of editing specific genes in the body which is responsible for a particular disease which is debilitating and by using a biological copy and paste technique: it can do everything from cut out HIV virus DNA to slow the growth of cancer cells and vice versa [2]. Any kind of disease where the body is deficient in specific molecules could potentially be targeted by this new technique. And if it works with diabetes, it could be time to say goodbye to needles and insulin injections. Any kind of disease where the body is deficient with specific molecules could potentially be targeted by this new technique. And if it works successfully with diabetes, it could be time to say goodbye to needles and insulin injections and also can be potentially be tried for the treatment of other disease like haemophilia and different cancers [3].

CRISPR-Cas9 editing of human embryos to correct an inherited form of heterotrophic cardiomyopathy (HCM) mutations in any one or multiple of these genes cause HCM [4]. Although autosomal dominant, these mutations face little selective pressure that might reduce their frequency because heart failure does not typically begin until after reproduction age. Mutations in MYBPC3 account for $40 \%$ of genetic HCM. The gene encodes the thick filamentassociated cardiac myosin-binding protein $\mathrm{C}$, which regulates structure of the sarcomeres in cardiac muscle [5]. This condition, however, is a common cause of sudden death among young athletes. The details of this work, published online August 2 in Nature, show the new methods avoid some of the pitfalls seen in prior attempts and would likely be safer [6]. Families with this form of HCM can use pre-implantation genetic diagnosis (PGD) after in vitro fertilization to select from the half of their embryos that do not inherit a parent's mutation [7]. If available, gene editing, which creates double-strand breaks in the DNA that permit replacement of the mutant allele with a normal (wild-type) one, would expand the pool of embryos from which to select.

Previous attempts, which introduced the gene editing (Cas9 enzyme and guide RNA) at fertilization, led to mosaic 8-celled embryos, in which some cells had the correction and some did not. Mosaic embryos would make PGD impossible and reproduction more, rather than less, risky for these couples with the new technique it completely possible to avoid mosaicism [8]. It can create fully corrected human embryos by shifting the timetable, injecting oocytes on the brink of fertilization, at metaphase of the second meiotic division, with sperm and the CRISPR components. In this sperms were from a man with a MYBPC3 4-base deletion mutation in one of the two copies of the gene (heterozygote); 12 healthy young women donated the oocytes, which had normal genes [9]. This new system used that copied wild-type gene from the oocyte. These oocytes provide the template for a natural form of DNA correction (homology-directed repair) that removes the mutant gene from the male genome and replaces it with a copy of the maternal normal gene. The DNA repair system, which is in the cytoplasm, comes from the oocyte because mature sperm have so little cytoplasm. So the introduction of CRISPR at the time of sperm injection eliminated the issue of mosaicism [10].

This new system comes with caution that, this approach for an autosomal dominant condition does not apply to an autosomal recessive condition, which is the mode of inheritance most common in young children with genetic diseases and these both copies of 
the targeted gene from an affected parent are mutant, an exogenous copy would need to be introduced, and that might not be safe. Adapting this technology to correct the two mutant alleles of an autosomal recessive condition could be exposing human gametes or embryos to small molecule inhibitors that could have deleterious effects on embryonic development [11].

\section{References}

1. Carrier L, Mearini G, Stathopoulou K, Cuello F (2015) Cardiac myosinbinding protein C (MYBPC3) in cardiac pathophysiology. Gene 573(2): 188-197.

2. Dhandapany PS, Sadayappan S, Xue Y, Powell GT, Rani DS, et al. (2009) A common MYBPC3 (cardiac myosin binding protein C) variant associated with cardiomyopathies in South Asia. Nat Genet 41(2): 187-191.

3. Struewing JP, Hartge P, Wacholder S, Baker SM, Berlin M, et al. (1997) The risk of cancer associated with specific mutations of BRCA1 and BRCA2 among Ashkenazi Jews. N Engl J Med 336(20): 1401-1408.

4. Schlossarek S, Mearini G, Carrier L (2011) Cardiac myosin-binding protein $\mathrm{C}$ in hypertrophic cardiomyopathy: mechanisms and therapeutic opportunities. J Mol Cell Cardiol 50(4): 613-620.
5. Kim S, Kim D, Cho SW, Kim J, Kim JS (2014) Highly efficient RNAguided genome editing in human cells via delivery of purified Cas9 ribonucleoproteins. Genome Res 24(6): 1012-1019.

6. Kang E, Wang X, Hedges R, Ma H, Folmes CD, et al. (2016) Age-related accumulation of somatic mitochondrial DNA mutations in adult-derived human iPSCs. Cell Stem Cell 18(5): 625-636.

7. Kim D, Kim S, Kim S, Park J, Kim JS (2016) Genome-wide target specificities of CRISPR-Cas9 nucleases revealed by multiplex Digenomeseq. Genome Res 26(11): 406-415.

8. Lange J, Yamada S, Tischfield SE, Pan J, Kim S, et al. (2016) The landscape of mouse meiotic double-strand break formation, processing, and repair Cell 167(3): 695-708.

9. Tang L, Zeng Y, Du H, Gong M, Peng J, et al. (2017) CRISPR/Cas9-mediated gene editing in human zygotes using Cas9 protein. Mol Genet Genomics 292(2): 525-533.

10. Tu Z, Yang W, Yan S, Yin A, Gao J, et al. (2017) Promoting Cas9 degradation reduces mosaic mutations in non-human primate embryos. Sci Rep 7: 42081.

11. Park J, Lim K, Kim JS, Bae S (2017) Cas-analyzer: an online tool for assessing genome editing results using NGS data. Bioinformatics 33(2): 286-288. 